\title{
Schisandrol B protects against acetaminophen- induced acute hepatotoxicity in mice via activation of the NRF2/ARE signaling pathway
}

\author{
Yi-ming JIANG ${ }^{1, \#}$, Ying WANG ${ }^{1,2, \#, ~ H u a-s e n ~ T A N ~}{ }^{1}$, Tao YU ${ }^{1}$, Xiao-mei FAN ${ }^{1}$, Pan $\mathrm{CHEN}^{1,3}$, Hang ZENG ${ }^{1}$, Min HUANG ${ }^{1, *}$, \\ Hui-chang $\mathrm{BI}^{1, *}$ \\ ${ }^{1}$ School of Pharmaceutical Sciences, Sun Yat-sen University, Guangzhou 510006, China; ${ }^{2}$ Sun Yat-sen Memorial Hospital, Sun Yat-sen \\ University, Guangzhou 510006, China; ${ }^{3}$ The First Affiliated Hospital, Sun Yat-sen University, Guangzhou 510006, China
}

Aim: The nuclear factor erythroid 2-related factor 2 (NRF2) acts through the antioxidant response element (ARE) to regulate the expression of many detoxifying and antioxidant genes responsible for cytoprotective processes. We previously reported that schisandrol B (SolB) isolated from Schisandra sphenanthera produced a protective effect against acetaminophen (APAP)-induced liver injury. In this study we investigated whether the NRF2/ARE signaling pathway was involved in this hepato-protective effect.

Methods: Male C57BL/ 6 mice were treated with SolB $\left(200 \mathrm{mg} \cdot \mathrm{kg}^{-1} \cdot \mathrm{d}^{-1}\right.$, ig) for $3 \mathrm{~d}$ before injection of APAP (400 mg/kg, ip). Serum and liver tissue samples were collected $6 \mathrm{~h}$ later. The mRNA and protein expression were measured using qRT-PCR and Western blot assay, respectively. The activation of NRF2 was examined in HepG2 cells using luciferase reporter gene assay.

Results: SolB pretreatment significantly alleviated the hepatic injury (large patchy necrosis and hyperemia of the hepatic sinus), the increase of serum AST, ALT levels and hepatic MDA contents, and the decrease of liver and mitochondrial glutathione levels in APAPtreated mice. Furthermore, SolB pretreatment significantly increased nuclear accumulation of NRF2 and increased hepatic expression of NRF2 downstream proteins, including GCLC, GSR, NQ01, GSTs, MRP2, MRP3 and MRP4 in APAP-treated mice. Moreover, treatment with SolB (2.5-20 $\mu \mathrm{mol} / \mathrm{L})$ dose-dependently increased the activity of NRF2 reporter gene in HepG2 cells.

Conclusion: SolB exhibits a remarkable protective effect against APAP-induced hepatotoxicity, partially via activation of the NRF2/ARE pathway and regulation of NRF2 target genes, which induce detoxification and increase antioxidant capacity.

Keywords: acetaminophen; hepatotoxicity; schisandrol B; Schisandra sphenanthera; liver injury; glutathione; NRF2; antioxidant resporse element

Acta Pharmacologica Sinica (2016) 37: 382-389; doi: 10.1038/aps.2015.120; published online 25 Jan 2016

\section{Introduction}

Acetaminophen (APAP) is the most commonly used analgesic and antipyretic drug in the world. Although it is safe at therapeutic doses, APAP overdoses frequently cause severe hepatotoxicity, which is an important public health concern worldwide ${ }^{[1]}$. APAP-induced acute liver injury is due to the formation of $N$-acetyl-p-benzoquinone imine (NAPQI), a highly electrophilic metabolite. Subsequently, NAPQI binds to cellular proteins and causes glutathione (GSH) depletion and oxidative stress, which may trigger signaling pathways that cause mitochondrial toxicity and ultimately result in lethal cell injury $^{[2,3]}$.

\footnotetext{
\#These authors contributed equally to this article.

* To whom correspondence should be addressed.

E-mail bihchang@mail.sysu.edu.cn (Hui-chang BI); huangmin@mail.sysu.edu.cn (Min HUANG)

Received 2014-12-10 Accepted 2015-08-25
}

Nuclear factor erythroid 2-related factor 2 (NRF2) acts through the antioxidant response element (ARE) to regulate the expression of many intracellular detoxifying and antioxidant genes that are responsible for cytoprotective processes, including GSH synthesis; antioxidative stress system activation; and conjugation, transport and excretion of toxic metabolites $^{[4]}$. The NRF2/ARE pathway has been identified as a protective response that is resistant to liver injury and plays an important role in alleviating toxicant-induced hepatotoxicity. Activation of the NRF2/ARE signaling pathway has been reported to protect animals against liver injuries caused by APAP and other hepatotoxicants ${ }^{[5]}$.

Schisandrol B (SolB) is one of the most important active components isolated from Schisandra sphenanthera, which is a wellknown herb that is widely used in China, Japan and Korea for its protective effects on liver, kidney, and heart ${ }^{[6]}$, especially for its effect against virus- and chemical-induced hepatitis ${ }^{[7,8]}$. Recently, we found that Wuzhi tablets (a preparation of an 
ethanolic extract of Schisandra sphenanthera) exert significant hepatic protection against APAP-induced liver damage. This effect was mediated by the inhibition of APAP bioactivation; activation of the NRF2/ARE pathway, which induces detoxification and antioxidation; and facilitation of liver regeneration following APAP-induced liver injury ${ }^{[9,10]}$. Most recently, we found that SolB is significantly hepato-protective against APAP-induced liver injury via inhibition of APAP bioactivation and regulation of liver regeneration ${ }^{[11]}$. Interestingly, we observed not only that co-treatment with SolB prevented APAP-induced decreases in GSH level, but also that administration of SolB alone increased GSH synthesis. These results indicate that SolB may exert an antioxidant effect via relevant signaling pathways, such as the NRF2/ARE pathway. Because SolB is one of the major active components in Wuzhi tablets, we assume that the NRF2/ARE signaling pathway may also play a role in SolB-mediated hepato-protection against APAPinduced toxicity. Therefore, this study aimed to investigate whether and how the NRF2/ARE signaling pathway contributes to the protective effect of SolB on APAP-induced toxicity.

\section{Materials and methods}

\section{Chemicals and reagents}

Schisandrol B (98\% pure) was purchased from Shanghai Winherb Medical Science and Technology Development Co, Ltd (Shanghai, China). APAP and other chemicals, unless otherwise indicated, were obtained from Sigma-Aldrich Chemical Co (St Louis, MO, USA).

Rabbit polyclonal anti-glutamate-cysteine ligase catalytic subunit (GCLC) and monoclonal anti-glutamate-cysteine ligase modifier subunit (GCLM) antibodies were obtained from Abcam (Abcam, Cambridge, UK). Rabbit monoclonal anti-multidrug resistance-associated protein 4 (MRP4), antihistone $\mathrm{H} 3$ and anti-glyceraldehyde-3-phosphate dehydrogenase (GAPDH) antibodies were all purchased from Cell Signaling Technology (Danvers, MA, USA). Rabbit polyclonal anti-NAD(P)H quinone oxidoreductase 1 (NQO1) and antiNRF2 antibodies and goat polyclonal anti-MRP2 and antiMRP3 antibodies were acquired from Santa Cruz Biotechnology (Santa Cruz, CA, USA). Rabbit polyclonal anti-glutathione synthetase (GSS), anti-glutathione reductase (GSR), antiglutathione S-transferase a (GST- $\alpha)$, anti-GST-ח, anti-GST- $\mu$, and anti-kelch-like ECH-associated protein 1 (KEAP1) antibodies were purchased from Sangon Biotechnology (Sangon Tech, Shanghai, China). Peroxidase-conjugated anti-rabbit and anti-mouse immunoglobulin G (IgG) were purchased from Cell Signaling Technology (Danvers, MA, USA). Anti-goat IgG was purchased from Santa Cruz Biotechnology (Santa Cruz, CA, USA).

The plasmid pGL3-ARE-Luc was an appreciated gift from Dr Athanassios FRAGOULIS (University Hospital Aachen, RWTH Aachen University, Germany) ${ }^{[12]}$. ARE refers to the ARE consensus sequence of the NQO1 promoter region. A dominant positive NRF2 expression vector ( $p E F-N R F 2$ ) was an appreciated gift from Dr Shinya ITO (The Hospital for Sick Children, Toronto, Canada) ${ }^{[13]}$.

\section{Experimental animals and treatment}

Male C57BL/6 mice weighing 20-22 g were provided by the Laboratory Animal Service Center of Sun Yat-sen University, Guangzhou, China. The animal room was maintained at $23 \pm 1{ }^{\circ} \mathrm{C}$ with a $12-\mathrm{h}$ light-dark cycle and $55 \% \pm 5 \%$ humidity. The mice were supplied with standard rodent chow and water ad libitum. The animal experiments described in this study were carried out in accordance with the Regulations of Experimental Animal Administration issued by the Ministry of Science and Technology of China (http://www.most.gov.cn). The protocol was approved by the Animal Ethics and Welfare Committee of Sun Yat-sen University.

The animal experimental design, treatment, and doses of APAP and SolB were similar to those described in our published report ${ }^{[11]}$. Briefly, 24 mice were randomly assigned to the following four groups: (1) Vehicle, (2) SolB (200 $\left.\mathrm{mg} \cdot \mathrm{kg}^{-1} \cdot \mathrm{d}^{-1}\right)$, (3) APAP (400 mg/kg)+Vehicle, and (4) APAP (400 mg/kg)+SolB $\left(200 \mathrm{mg} \cdot \mathrm{kg}^{-1} \cdot \mathrm{d}^{-1}\right)$. APAP was dissolved in a saline solution, and SolB was suspended in a $0.5 \%(w / v)$ sodium carboxymethyl cellulose (CMC-Na) solution. All mice in groups 2 and 4 were gavaged with SolB solution seven times at intervals of $12 \mathrm{~h}$. The APAP treatment was administered to the mice as a single intraperitoneal injection at a dose of $400 \mathrm{mg} / \mathrm{kg}$, and the untreated vehicle group and SolB-only group were intraperitoneally injected with saline solution. For the APAP group and SolB/APAP group, the APAP was administered $15 \mathrm{~min}$ after the last gavage treatment. The mice in the vehicle control group received an ip injection of saline and were gavaged with $0.5 \%$ CMC-Na. Blood and livers were harvested $6 \mathrm{~h}$ after the APAP treatment. The blood was collected after removal of an eyeball. Serum samples were isolated from the blood by centrifugation at $3000 \times g$ for $10 \mathrm{~min}$. A portion of each liver was immediately fixed in $10 \%$ buffered formalin for histological sectioning, and the remaining tissues were flash frozen in liquid nitrogen and stored at $-80^{\circ} \mathrm{C}$ for further use.

\section{Histological and biochemical assessment}

Histological and biochemical assessments were performed according to standard protocols as described in our previously published reports ${ }^{[10,14]}$. The liver tissues fixed in neutral buffered formalin were embedded in paraffin, cut into 3- $\mu$ m-thick sections, and stained with hematoxylin and eosin (H\&E). The H\&E-stained liver sections were evaluated using a LEICA DM5000B Microscope (Leica, Heidelberg, Germany). Serum levels of alanine transaminase (ALT) and aspartate transaminase (AST) were determined to evaluate liver injury using a Beckman Synchron CX5 Clinical System and a commercial reagent kit (Kefang Biotech, Guangzhou, China). Reduced glutathione (GSH) levels in the liver and liver mitochondrial extracts were measured using a commercially available assay kit (Nanjing Jiancheng Bioengineering Institute, Nanjing, China). Hepatocyte mitochondria were isolated by differential centrifugation following the manufacturer's instructions (Sangon Tech, Shanghai, China). Malondialdehyde (MDA) levels in the liver were assessed using a commercially available assay kit (Nanjing Jiancheng Bioengineering Institute, Nanjing, China). 


\section{Quantitative real-time PCR analysis of NRF2}

Total RNA was isolated from the mouse liver samples using TRIzol reagent according to the manufacturer's instruction (Invitrogen, Grand Island, NY, USA) and quantified using a NanoDrop spectrophotometer (Thermo Scientific, Rockford, IL, USA) as described in a previous report ${ }^{[15]}$. cDNA synthesis was performed with $1 \mu \mathrm{g}$ of total RNA using a PrimeScript RT reagent kit with gDNA eraser (TaKaRa Biotech, Kyoto, Japan). Quantitative real-time PCR (qRT-PCR) analysis was performed using a SYBR Premix Ex Taq II kit (TaKaRa Biotech, Kyoto, Japan) in an ABI 7500 Sequence Detection System (Applied Biosystems, Foster City, CA, USA). GAPDH was analyzed in each sample to normalize expression. Gene-specific primers were obtained from a primer bank, and the primer sequences used in this study were as follows: NRF2 forward primer (5'-CGAGATATACGCAGGAGAGGTAAGA-3') and reverse primer (5'-GCTCGACAATGTTCTCCAGCTT-3'). GAPDH forward primer (5'-AGGTCGGTGTGAACGGATTTG-3') and reverse primer (5'-GGGGTCGTTGATGGCAACA-3').

\section{Western blot analysis}

Western blot analyses were performed as described in our previous reports ${ }^{[1,15]}$. Briefly, protein extracts were prepared from the liver tissue using RIPA lysis buffer (Biocolors, Shanghai, China) or a Nuclear Extract kit (Active Motif, Carlsbad, CA, USA) according to the manufacturer's instructions. Protein concentrations were determined using a Bicinchoninic Acid protein assay (Thermo Scientific, Rockford, IL, USA). A 40- $\mu \mathrm{g}$ sample of each of the protein extracts was separated on an $8 \%-15 \%$ SDS gel via SDS-PAGE and transferred to a polyvinylidene fluoride membrane (Millipore, Bedford, USA). Next, the membranes were blocked in Tris-buffered saline containing $5 \%$ BSA or $5 \%$ nonfat dry milk for $1 \mathrm{~h}$ at room temperature and then incubated with primary antibody overnight at $4{ }^{\circ} \mathrm{C}$. The proteins were visualized using an electrochemiluminescence (ECL) detection kit (Engreen Biosystem, China). Protein band intensities were analyzed using Quantity One software (Bio-Rad Laboratories, Hercules, USA).

\section{Cell culture and cell viability assay}

HepG2 cells (ATCC, Manassas, VA, USA) were cultured in DMEM containing 10\% FBS and $100 \mathrm{U} / \mathrm{mL}$ penicillin/streptomycin at $37{ }^{\circ} \mathrm{C}$ in $5 \% \mathrm{CO}_{2}$. The effect of SolB on cell viability was measured using an MTT assay. Briefly, HepG2 cells at $1 \times 10^{5}$ cell $/ \mathrm{mL}$ were seeded in 96 -well plates, $100 \mu \mathrm{L}$ of SolB at various concentrations $(2.5,5,10,20,40,80$, or $160 \mu \mathrm{mol} / \mathrm{L})$ was added, and the cells were incubated for $24 \mathrm{~h}$. After this incubation, cell viability was assessed by adding $10 \mu \mathrm{L} /$ well of MTT (5 mg/mL) and incubating for $4 \mathrm{~h}$. Then, the supernatants of the cultures were removed and replaced with $100 \mu \mathrm{L}$ DMSO. The cell viability rate (\%) was calculated as the absorbance of the SolB-treated cells divided by the absorbance of the untreated control cells.

\section{Transient transfection assays}

HepG2 cells were plated at a density of $1.5 \times 10^{4}$ cells/well in 96-well plates $24 \mathrm{~h}$ before transfection. The cells were cotransfected with $50 \mathrm{ng}$ pEF-NRF2, $100 \mathrm{ng}$ pGL3-ARE-Luc and 5 ng of the Renilla luciferase reporter plasmid pRL-TK using Lipofectamine 2000 reagent (Invitrogen, Grand Island, NY, USA) according to the manufacturer's instructions. Six hours after transfection, the cells were treated for $24 \mathrm{~h}$ with SolB (2.5, $5,10$, and $20 \mu \mathrm{mol} / \mathrm{L})$ or the agonist sulforaphane (SFN) (10 $\mu \mathrm{mol} / \mathrm{L})$ as a positive control. Cell lysates were then prepared, and luciferase activity was measured in a luminometer using the Dual Reporter Assay System (Promega, Madison, WI, USA) according to the manufacturer's protocol. Firefly luciferase activity was normalized to Renilla luciferase activity for each well.

\section{Statistical analysis}

The data are expressed as the meantstandard error of the mean (SEM). Significant differences were analyzed using Oneway ANOVA followed by an unpaired Student's $t$-test or Dunnett's multiple comparison post hoc test using GraphPad Prism 5 (GraphPad Software Inc, San Diego, CA, USA). Differences were considered significant at $P$ values less than 0.05 .

\section{Results}

\section{SolB protects against APAP-induced hepatotoxicity in mice}

As expected, the mice given intraperitoneal doses of APAP (400 mg/kg) exhibited typical histopathological signs of APAP-induced hepatotoxicity, including large patchy necrosis and hyperemia of the hepatic sinus. This pattern was significantly attenuated by pretreatment with SolB (Figure 1A). At a magnification of $400 \times$, the APAP-treated mice displayed obvious centrilobular hepatic necrosis and focal intrahepatic hemorrhage around the central venous lesions, whereas the mice co-treated with SolB and APAP showed normal liver histology (Figure 1B). In addition, their serum biochemical parameters were consistent with the liver morphological and histopathological results. ALT and AST activities markedly increased

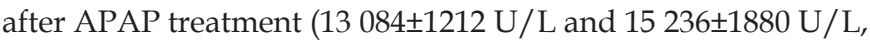
respectively). In contrast, pretreatment with SolB reduced the APAP-induced increase of ALT to $11 \%$ and of AST to $16 \%$ (Figure 2A and 2B). Furthermore, APAP (400 mg/kg) resulted in a decrease in the total liver and mitochondrial GSH levels, while SolB pretreatment increased total hepatic GSH to 2.1-fold and increased mitochondrial GSH levels to 1.9-fold (Figure 2C and 2D). The hepatic MDA contents reflected an increase in oxidative stress. The MDA level was increased to 1.7-fold in the APAP-treated mice compared to the vehicletreated mice. Hepatic MDA was significantly suppressed by pretreatment with SolB (Figure 2E). Taken together, these data clearly illustrate that SolB can protect against APAP-induced liver damage, possibly by decreasing GSH depletion and oxidative stress.

\section{Effect of SolB on NRF2 mRNA level and nuclear translocation}

To examine whether SolB affects NRF2 signaling, mRNA expression and nuclear translocation of NRF2 were measured in mice. Figure $3 \mathrm{~A}$ shows that the expression level of NRF2 
was slightly changed by APAP treatment. Treatment with SolB alone increased NRF2 mRNA expression, and SolB/APAP co-treatment further enhanced this SolB-mediated increase. Furthermore, SolB markedly increased the nuclear translocation of NRF2 (SolB alone caused a 0.4-fold increase, and SolB/
APAP co-treatment caused a 0.8-fold increase in nuclear NRF2 compared to the APAP group) (Figure 3B and 3C). However, the expression level of cytoplasmic KEAP1 was significantly decreased by the SolB treatment compared to the APAP group (Figure 3D and 3E), also indicating that SolB enhanced the
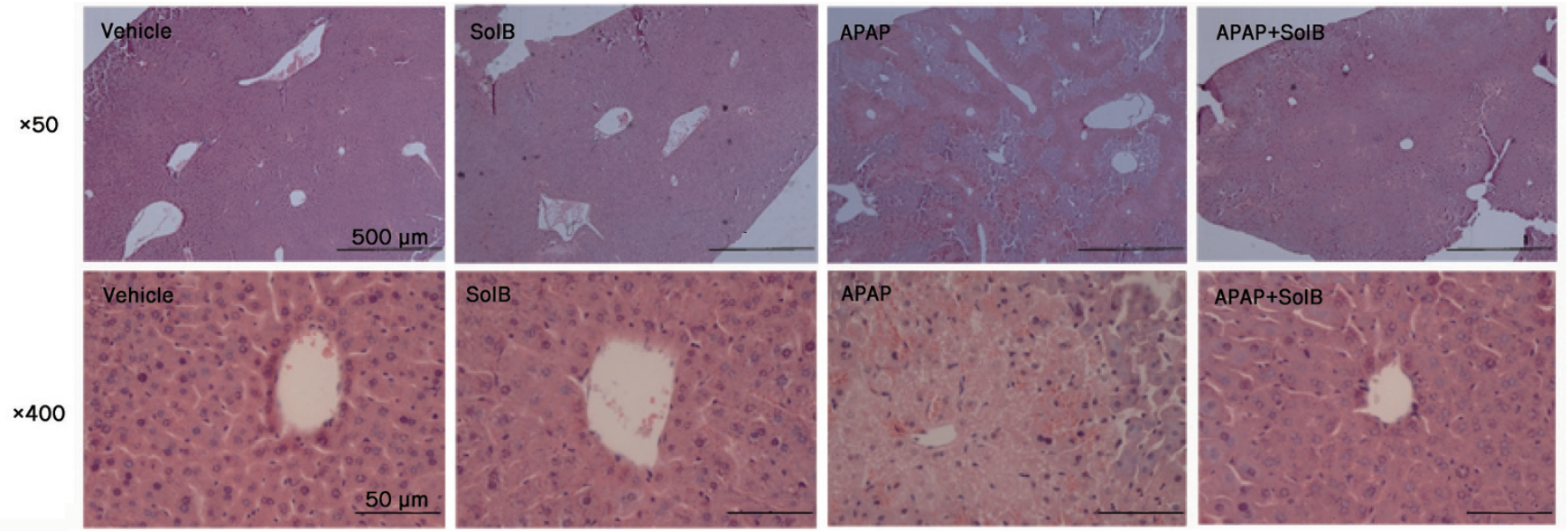

Figure 1. Histopathological analysis of representative H\&E-stained mouse liver samples. From the left: Vehicle; SolB (200 mg/kg); APAP (400 $\mathrm{mg} / \mathrm{kg})+$ Vehicle; APAP $(400 \mathrm{mg} / \mathrm{kg})+$ SolB $(200 \mathrm{mg} / \mathrm{kg})$.
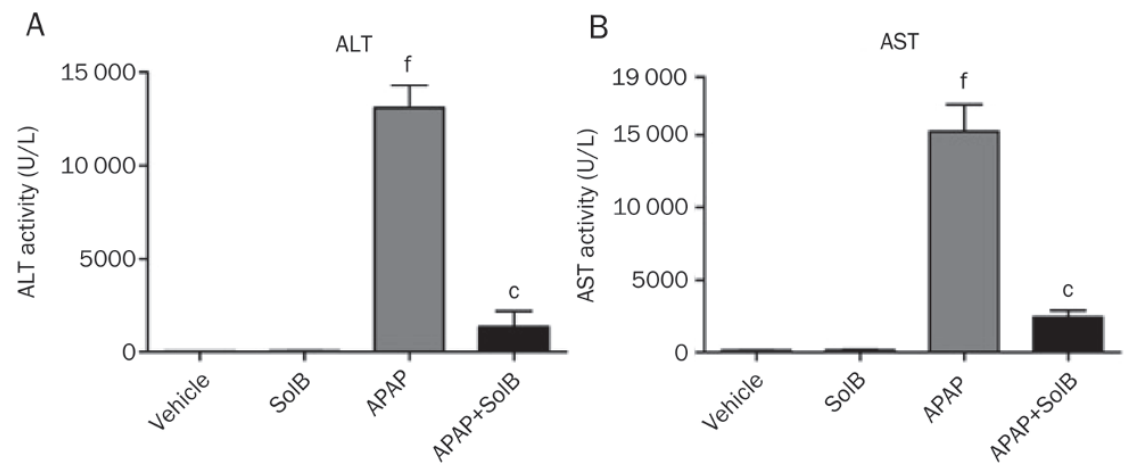

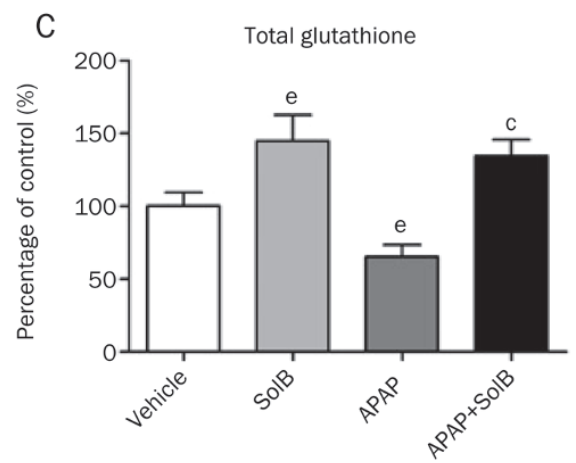

D

E

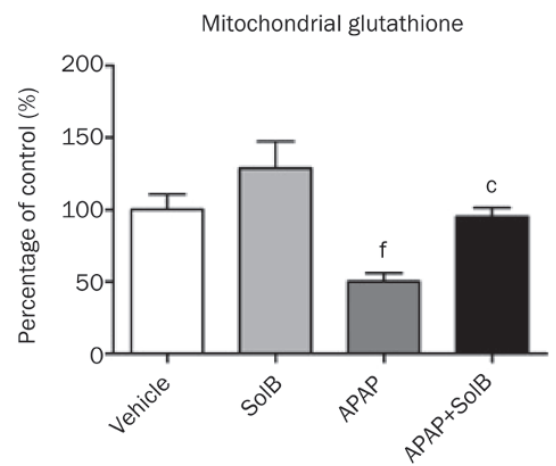

Total malondialdehyde

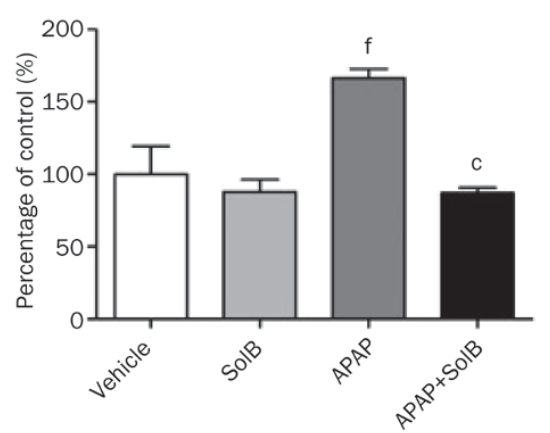

Figure 2. Biochemical assessment of AST, ALT, total GSH, mitochondrial GSH and total MDA $6 \mathrm{~h}$ after APAP treatment. Control and SolB pre-treated mice were treated with a hepatotoxic dose of APAP or left untreated, and blood or liver samples were analyzed after $6 \mathrm{~h}$ for AST, ALT, total GSH, mitochondrial GSH and total MDA. (A-B) Serum ALT and AST activities. (C-D) Total and mitochondrial GSH levels. (E) Total MDA levels. The data are expressed as the mean \pm SEM $(n=6)$. ${ }^{\mathrm{C}} P<0.01$ compared to the APAP group; ${ }^{\mathrm{e}} \mathrm{P}<0.05,{ }^{\mathrm{f}} \mathrm{P}<0.01$ compared to the vehicle group. 
nuclear translocation of NRF2.

Effect of SolB on the expression of NRF2 target proteins in the liver In this study, the target proteins of NRF2 that are essential for glutathione synthesis, including GSR, GSS, GCLC and GCLM, were examined by Western blotting. As shown in Figure $4 \mathrm{~A}-4 \mathrm{E}$, treatment with SolB alone significantly increased the protein expression of GSR and GCLC, and SolB/APAP cotreatment showed a similar effect on these proteins (1.2- and 0.8 -fold higher than that of the APAP group, respectively). No significant changes in GSS and GCLM protein levels were observed after APAP or SolB treatment. Furthermore, GST-a, GST- $\mu$, GST-ח and NQO1, which serve to detoxify reactive intermediates, were examined. The SolB treatment resulted in 3.1-, 2.5-, and 2.6-fold elevations in GST- $\alpha$, GST- $\mu$ and NQO1, and the SolB/APAP co-treatment caused 1.8-, 2.0-, and 2.2-fold increases, but no changes in the level of GST-п were observed (Figure 4A, 4F-4I). The MRP2, MRP3 and MRP4 are also regulated by NRF2 and are important in mediating the detoxification of APAP in the liver ${ }^{[16,17]}$. SolB markedly increased the expression of MRP2, MRP3 and MRP4 (1.4-, 1.2- and 1.1-fold higher than that of the vehicle) (Figure 4A, $4 \mathrm{~K}-4 \mathrm{M}$ ). APAP alone also markedly increased these MRPs to 2.6-, 2.0- and 2.5fold, which was likely a compensatory reaction due to liver injury. Co-treatment with APAP and SolB induced dramatic increase in MRP2, MRP3 and MRP4 levels, enhancing them to 2.7-, 2.7- and 4.4-fold, respectively. Taken together, these data indicated that the hepatoprotective effect of SolB against
APAP-induced liver toxicity may partially be the result of SolB-induced activation of the NRF2 signaling pathway, which resulted in enhanced detoxification and antioxidant capacities.

\section{Effect of SolB on NRF2 activation in HepG2 cells}

The effect of SolB on HepG2 cell viability was evaluated using an MTT assay. It was found that SolB was not toxic to HepG2 cells, even at concentrations up to $160 \mu \mathrm{mol} / \mathrm{L}$. The effect of SolB on NRF2 activation was further examined using luciferase reporter assays in HepG2 cells transiently transfected with reporter plasmids. Sulforaphane (SFN), a typical NRF2 activator, increased the luciferase activity of the NRF2 reporter gene to 2.8-fold compared to the vehicle-treated cells, whereas SolB dose-dependently increased the luciferase activity of the NRF2 reporter gene to 1.2-, 1.5-, 2.5- and 2.6-fold at 2.5, 5, 10 and 20 $\mu \mathrm{mol} / \mathrm{L}$, respectively (Figure 5). These data suggest that SolB is a NRF2 activator and contributes to the regulation of NRF2 target proteins that improve detoxification and antioxidant capacities and thus provides hepatoprotection against APAPinduced toxicity.

\section{Discussion}

APAP has become the most common cause of drug-induced liver injury, which is an important public health concern worldwide. Currently, $N$-acetylcysteine (NAC) is the primary antidote for APAP-induced hepatotoxicity in clinical practice, but its efficacy is limited to the early stages of APAP toxicity ${ }^{[18]}$. Previously, we found that SolB significantly protects against

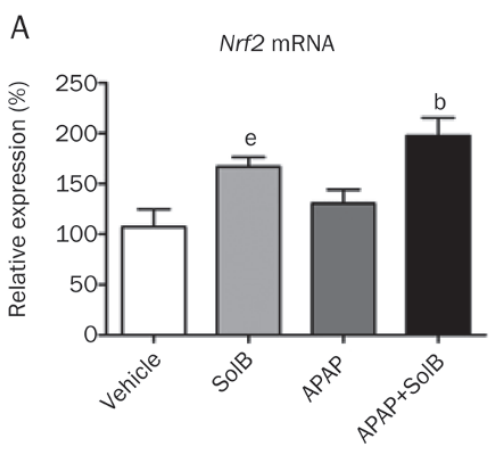

B
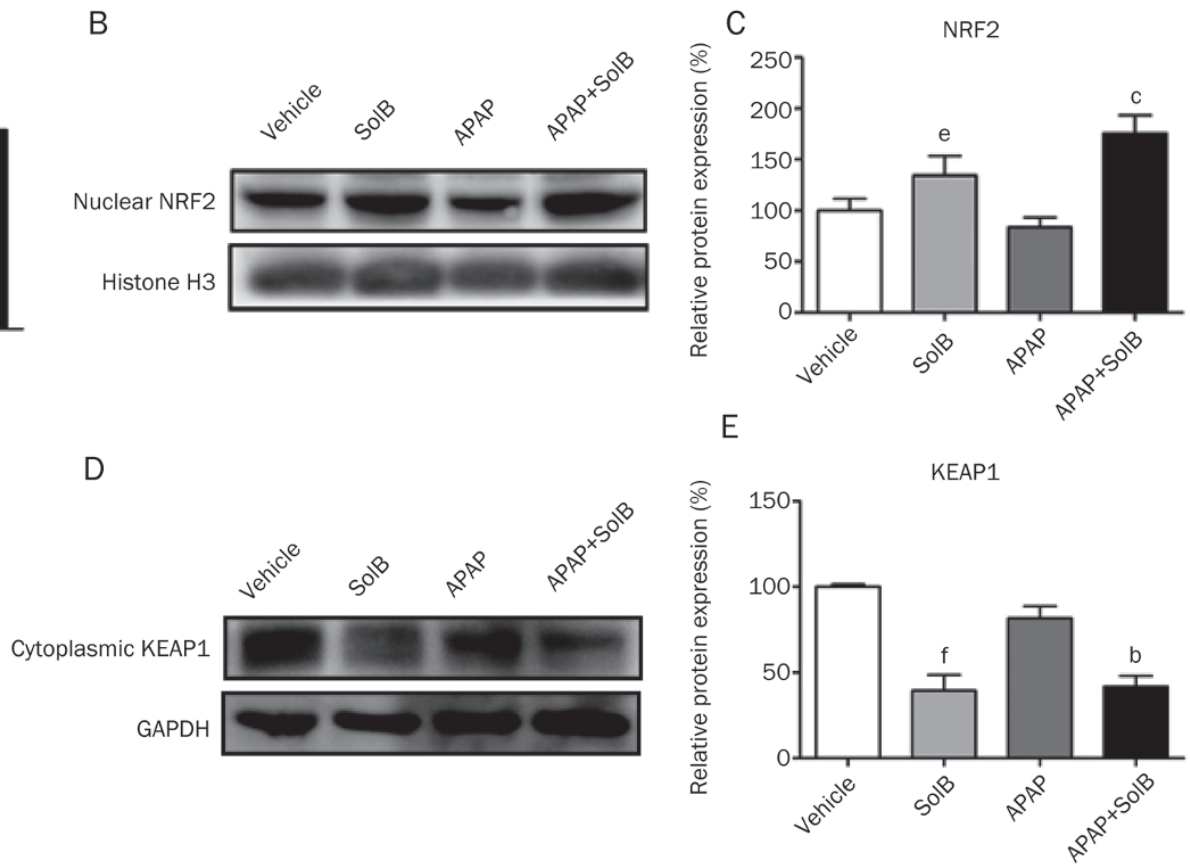

Figure 3. The effects of SolB on activation and expression of NRF2 in vivo. (A) qRT-PCR analysis was performed to measure the gene expression of $\mathrm{Nrf2}$, and the data are expressed as the mean \pm SEM $(n=6)$. (B-C) Western blotting was used to measure nuclear NRF2 protein expression. Specific band intensities were quantified and normalized to histone $\mathrm{H} 3$ and expressed as the mean $\pm \mathrm{SEM}(n=3)$. (D-E) Western blotting was used to measure cytoplasmic KEAP1 protein expression. Specific band intensities were quantified and normalized to GAPDH and expressed as the mean \pm SEM ( $n=3$ ). ${ }^{\mathrm{b}} P<0.05,{ }^{\mathrm{c}} P<0.01$ compared to the APAP group; ${ }^{\mathrm{e}} P<0.05,{ }^{\mathrm{f}} P<0.01$ compared to the vehicle group. 
A

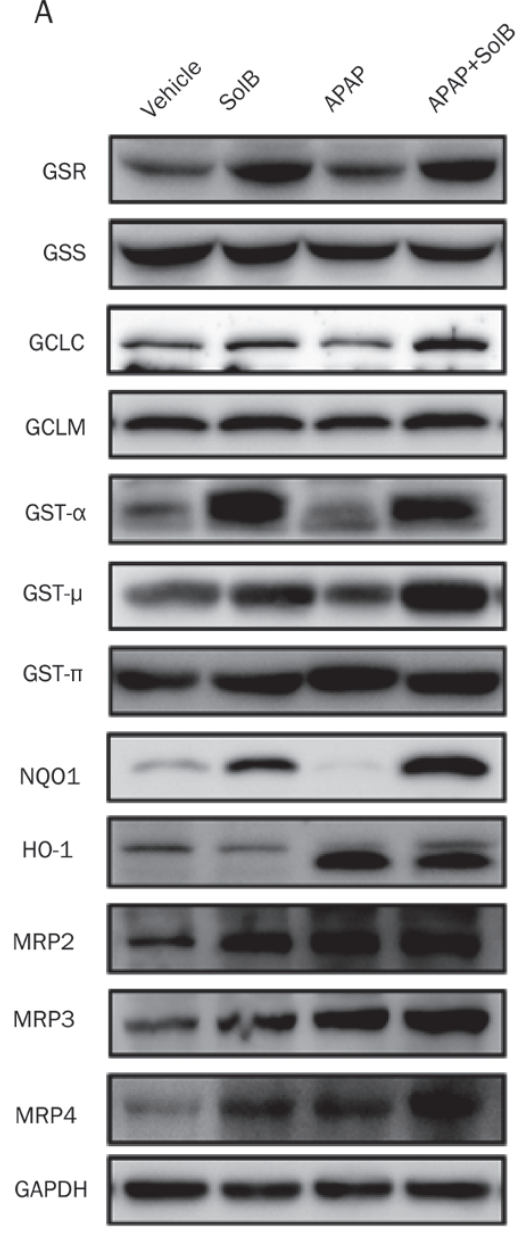

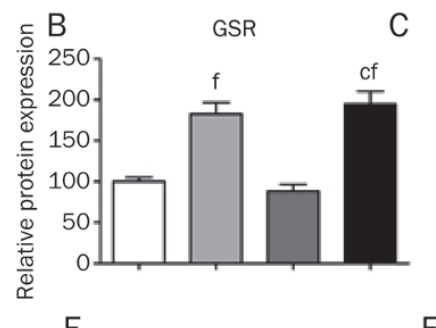
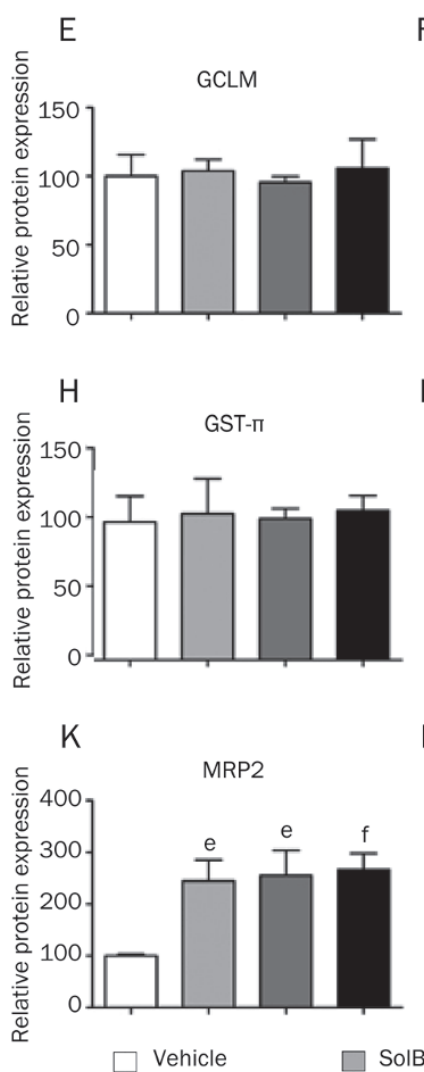

I

L

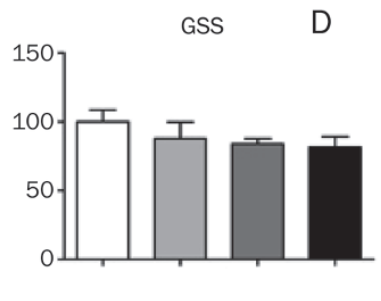

F

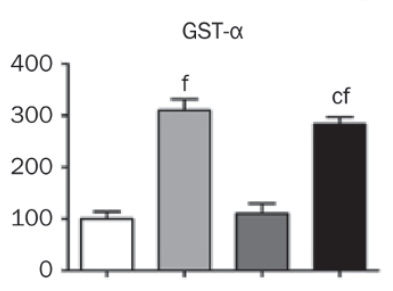

G
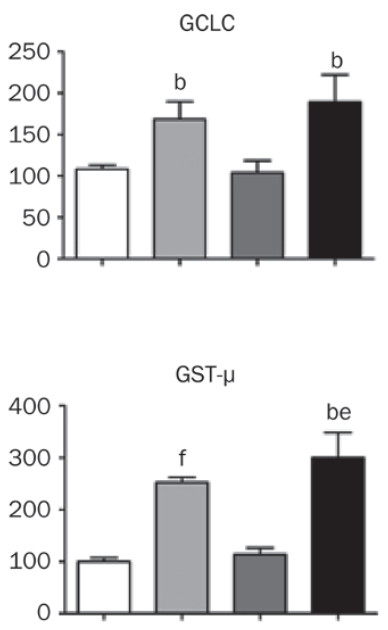

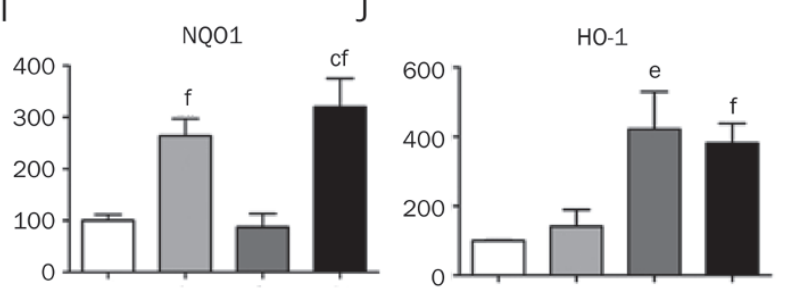

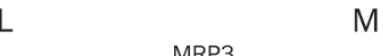

M

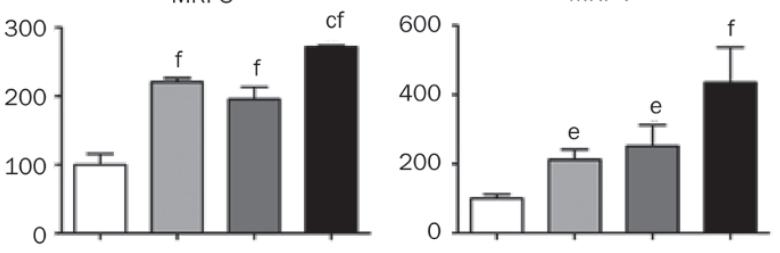

APAP+SoIB

Figure 4. Role of NRF2 signaling and its downstream proteins in SolB-mediated protection against APAP-induced liver toxicity. (A) Western blotting was used to measure the protein expression of GSR, GSS, GCLC, GCLM, GST- $\alpha$, GST- $\mu$, GST-п, NQ01, HO-1, MRP2, MRP3 and MRP4. (B-M) Specific band intensities were quantified and normalized to GAPDH. The data are expressed as the mean \pm SEM $(n=3) .{ }^{b} P<0.05,{ }^{\circ} P<0.01$ compared to the APAP group; ${ }^{\mathrm{e}} \mathrm{P}<0.05,{ }^{\mathrm{f}} \mathrm{P}<0.01$ compared to the vehicle group.
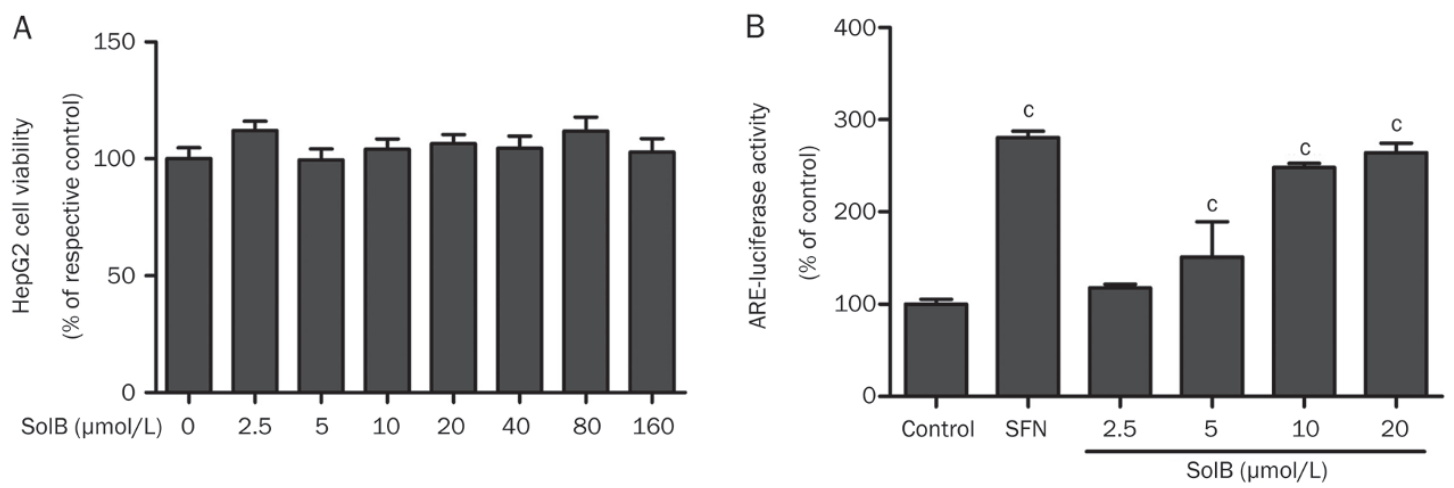

Figure 5. Effects of SolB on cell viability and NRF2 activation. (A) An MTT assay was used to measure HepG2 cell viability after treatment with SolB at 2.5 to $160 \mu \mathrm{mol} / \mathrm{L}$. (B) A luciferase reporter assay was used to measure the effect of SolB on NRF2 activation. The HepG2 cells were transiently transfected with plasmids as described in the Materials and methods section, and $6 \mathrm{~h}$ later the cells were treated with various concentrations of SolB (2.5-20 $\mu$ mol/L) or the positive agonist SFN $(10 \mu \mathrm{mol} / \mathrm{L})$ for $24 \mathrm{~h}$. The data are expressed as the mean \pm SEM $(n=5) .{ }^{c} P<0.01$ compared to the control. 
APAP-induced liver injury potentially via the inhibition of APAP bioactivation and regulation of liver regeneration ${ }^{[11,19]}$. However, whether and how the NRF2/ARE signaling pathway contributes to the protective effect of SolB against APAPinduced hepatotoxicity remained unknown. The present data clearly demonstrated that SolB is hepatoprotective against APAP-induced liver injury as indicated by morphological and histological assessment, as well as by biochemical parameters, including AST, ALT, GSH and MDA levels. Furthermore, the current study clearly demonstrates that SolB is a NRF2 activator and that this activation plays an important role in SolBmediated hepatoprotection against APAP-induced liver toxicity. Thus, these data clearly illustrate the contribution of the NRF2/ARE pathway to the hepato-protective effect of SolB against APAP-induced toxicity.

The importance of the NRF2 pathway in the regulation of GSH synthesis and cellular detoxification has been previously highlighted ${ }^{[20-22]}$. SolB can significantly attenuate APAPinduced hepatotoxicity and prevent changes in MDA and GSH levels. Furthermore, SolB enhanced nuclear NRF2 translocation and elevated the luciferase activity of an NRF2 reporter gene, which suggests that SolB exerts a beneficial effect by enhancing NRF2-regulated antioxidant and detoxification systems, thus offering protection against APAP-induced hepatotoxicity.

Currently, increasing attention has been paid to potential drugs that may act as therapeutic agents against APAPinduced hepatotoxicity by restoring or maintaining hepatic GSH content ${ }^{[23]}$. A genome-wide association study demonstrated that the GSH pathway is partially associated with variations in NAPQI toxicity ${ }^{[24]}$. A possible explanation for the prevention of NAPQI-induced GSH depletion is the facilitation of GSH biosynthesis. As mentioned previously, the NRF2-targeted GCLC and GSR genes are rate-limiting enzymes in GSH synthesis. The observed induction of these two proteins by SolB was accompanied by an increase in GSH levels, which may explain how SolB/APAP co-treatment was able to increase GSH levels and how SolB alone resulted in an abundance of GSH. These observations provide evidence that upregulation of GCLC and GSR by SolB through the NRF2/ ARE pathway resulted in an increase in GSH synthesis.

Furthermore, SolB significantly induced antioxidant defense and conjugation proteins, including NQO1, GST- $\alpha$ and GST- $\mu$. GSTs catalyze the conjugation of GSH with a series of electrophiles, including NAPQI ${ }^{[4]}$. The metabolites produced by glutathionylation are more hydrophilic, typically less reactive and readily excreted ${ }^{[25]}$. Moreover, recent studies have indicated that NQO1 possesses the capacity to enzymatically reduce NAPQI, which ameliorates APAP-induced toxicity ${ }^{[26]}$. Therefore, the induction of GSTs and NQO1 by SolB also contributes to the protection provided by SolB against APAP-induced hepatotoxicity.

In addition to enhancing hepatic detoxification and GSH levels, NRF2 stimulates the expression of MRP transporters, which transport and excrete metabolites from the liver. MRP2 transports xenobiotics and their glucuronate- or sulfate- conjugated metabolites into the bile or urine, whereas MRPs in the basolateral membrane transport these metabolites into the bloodstream for renal excretion ${ }^{[17,27]}$. In this study, SolB and APAP treatment were demonstrated to orchestrate adaptive responses by increasing MRP2, MRP3, and MRP4, and expression of these MRPs was further increased by SolB/APAP cotreatment. The induction of MRP2, MRP3, and MRP4 may contribute to the hepatoprotection provided by SolB consisting of enhancing the excretion of reactive or conjugated metabolites.

In summary, this study clearly demonstrated that SolB can protect against APAP-induced liver injury, and its hepatoprotective effect is partially due to activation of the NRF2-ARE signaling pathway, which regulates GSH synthesis, conjugation and excretion, enhancing antioxidant and detoxification capacity.

\section{Acknowledgements}

This study was financially supported by the National Natural Science Foundation of China (№ 81373470 and 81320108027), the Major Scientific and Technological Project (No 2012ZX09303012-002 and 2011A080300001), Guangdong Provincial Key Laboratory and Natural Science Foundation of Guangdong Province (No 2011A060901014 and S2012030006240), and the National Science and Technology Support Plan Projects (№ 2012BAI29B09).

\section{Author contribution}

Min HUANG, Hui-chang BI and Yi-ming JIANG participated in experimental design; Yi-ming JIANG, Ying WANG and Hua-sen TAN performed the experiments; Ying WANG and Xiao-mei FAN contributed new reagents and analytic tools; Yi-ming JIANG, Pan CHEN and Hang ZENG performed data analysis; and Yi-ming JIANG, Hui-chang BI and Tao YU contributed to the writing or revision of the manuscript.

\section{Abbreviations}

APAP, acetaminophen; SolB, schisandrol B; NAPQI, N-acetylp-benzoquinone imine; ALT, alanine aminotransferase; AST, aspartate aminotransferase; GSH, glutathione; NRF2, nuclear factor erythroid 2-related factor 2; ARE, antioxidant response element; SFN, Sulforaphane; NQO1, NAD(P)H quinone oxidoreductase 1; GCLC, glutamate-cysteine ligase catalytic subunit; GCLM, glutamate-cysteine ligase modifier subunit; MRP, multidrug resistance-associated protein; GAPDH, glyceraldehyde3-phosphate dehydrogenase; GSS, glutathione synthetase; GSR, glutathione reductase; KEAP1, kelch-like ECH-associated protein 1; GST, glutathione $S$-transferase; SFN, sulforaphane.

\section{References}

1 Dargan PI, Jones AL. Acetaminophen poisoning: an update for the intensivist. Crit Care 2002; 6: 108-10.

2 Ghosh J, Das J, Manna P, Sil PC. Arjunolic acid, a triterpenoid saponin, prevents acetaminophen (APAP)-induced liver and hepatocyte injury via the inhibition of APAP bioactivation and JNK-mediated mitochondrial protection. Free Radic Biol Med 2010; 48: 535-53. 
3 McGill MR, Sharpe MR, Williams CD, Taha M, Curry SC, Jaeschke H. The mechanism underlying acetaminophen-induced hepatotoxicity in humans and mice involves mitochondrial damage and nuclear DNA fragmentation. J Clin Invest 2012; 122: 1574-83.

4 Gum SI, Cho MK. Recent updates on acetaminophen hepatotoxicity: the role of nrf2 in hepatoprotection. Toxicol Res 2013; 29: 165-72.

5 Enomoto A, Itoh K, Nagayoshi E, Haruta J, Kimura T, O'Connor T, et al. High sensitivity of Nrf2 knockout mice to acetaminophen hepatotoxicity associated with decreased expression of ARE-regulated drug metabolizing enzymes and antioxidant genes. Toxicol Sci 2001; 59: 169-77.

6 Panossian A, Wikman G. Pharmacology of Schisandra chinensis Bail: an overview of Russian research and uses in medicine. J Ethnopharmacol 2008; 118: 183-212.

7 Teraoka R, Shimada T, Aburada M. The molecular mechanisms of the hepatoprotective effect of gomisin A against oxidative stress and inflammatory response in rats with carbon tetrachloride-induced acute liver injury. Biol Pharm Bull 2012; 35: 171-7.

8 Zhu M, Yeung RY, Lin KF, Li RC. Improvement of phase I drug metabolism with Schisandra chinensis against $\mathrm{CCl}_{4}$ hepatotoxicity in a rat model. Planta Med 2000; 66: 521-5.

9 Bi H, Li F, Krausz KW, Qu A, Johnson CH, Gonzalez FJ. Targeted metabolomics of serum acylcarnitines evaluates hepatoprotective effect of Wuzhi tablet (Schisandra sphenanthera extract) against acute acetaminophen toxicity. Evid Based Complement Alternat Med 2013; 2013: 985257.

10 Fan $\mathrm{X}$, Jiang $\mathrm{Y}$, Wang $\mathrm{Y}$, Tan $\mathrm{H}$, Zeng $\mathrm{H}$, Wang $\mathrm{Y}$, et al. Wuzhi tablet (Schisandra sphenanthera extract) protects against acetaminopheninduced hepatotoxicity by inhibition of CYP-mediated bioactivation and regulation of NRF2-ARE and p53/p21 pathways. Drug Metab Dispos 2014; 42: 1982-90.

11 Jiang $\mathrm{Y}$, Fan $\mathrm{X}$, Wang $\mathrm{Y}$, Chen $\mathrm{P}$, Zeng $\mathrm{H}$, Tan $\mathrm{H}$, et al. Schisandrol $\mathrm{B}$ protects against acetaminophen-induced hepatotoxicity by inhibition of CYP-mediated bioactivation and regulation of liver regeneration. Toxicol Sci 2015; 143: 107-15.

12 Fragoulis A, Laufs J, Muller S, Soppa U, Siegl S, Reiss LK, et al. Sulforaphane has opposing effects on TNF-alpha stimulated and unstimulated synoviocytes. Arthritis Res Ther 2012; 14: R220.

13 Tan KP, Kosuge L, Yang MD, Ito SY. NRF2 as a determinant of cellular resistance in retinoic acid cytotoxicity. Free Radic Biol Med 2008; 45: 1663-73.

14 Shackelford C, Long G, Wolf J, Okerberg C, Herbert R. Qualitative and quantitative analysis of nonneoplastic lesions in toxicology studies. Toxicol Pathol 2002; 30: 93-6.

15 Chen P, Zeng H, Wang Y, Fan X, Xu C, Deng R, et al. Low dose of oleanolic acid protects against lithocholic acid-induced cholestasis in mice: potential involvement of nuclear factor-E2-related factor 2-mediated upregulation of multidrug resistance-associated proteins. Drug Metab Dispos 2014; 42: 844-52.

16 Gum SI, Cho MK. The amelioration of $N$-acetyl-p-benzoquinone imine toxicity by ginsenoside Rg3: the role of Nrf2-mediated detoxification and Mrp1/Mrp3 transports. Oxid Med Cell Longev 2013; 2013: 957947.

17 Aleksunes LM, Slitt AL, Maher JM, Augustine LM, Goedken MJ, Chan JY, et al. Induction of Mrp3 and Mrp4 transporters during acetaminophen hepatotoxicity is dependent on Nrf2. Toxicol Appl Pharmacol 2008; 226: 74-83.

18 Yip L, Dart RC. A 20-hour treatment for acute acetaminophen overdose. N Engl J Med 2003; 348: 2471-2.

19 Jiang $\mathrm{Y}$, Fan X, Wang Y, Tan $\mathrm{H}$, Chen $\mathrm{P}$, Zeng $\mathrm{H}$, et al. Hepato-protective effects of six schisandra lignans on acetaminophen-induced liver injury are partially associated with the inhibition of CYP-mediated bioactivation. Chem Biol Interact 2015; 231: 83-9.

20 Chan K, Han XD, Kan YW. An important function of Nrf2 in combating oxidative stress: detoxification of acetaminophen. Proc Natl Acad Sci U S A 2001; 98: 4611-6.

21 Goldring CE, Kitteringham NR, Elsby R, Randle LE, Clement YN, Williams DP, et al. Activation of hepatic Nrf2 in vivo by acetaminophen in CD-1 mice. Hepatology 2004; 39: 1267-76.

22 Patterson AD, Carlson BA, Li F, Bonzo JA, Yoo MH, Krausz KW, et al. Disruption of thioredoxin reductase 1 protects mice from acute acetaminophen-induced hepatotoxicity through enhanced NRF2 activity. Chem Res Toxicol 2013; 26: 1088-96.

23 Saberi B, Ybanez MD, Johnson HS, Gaarde WA, Han D, Kaplowitz N. Protein kinase $\mathrm{C}(\mathrm{PKC})$ participates in acetaminophen hepatotoxicity through c-jun-N-terminal kinase (JNK)-dependent and -independent signaling pathways. Hepatology 2014; 59: 1543-54.

24 Moyer AM, Fridley BL, Jenkins GD, Batzler AJ, Pelleymounter LL, Kalari $\mathrm{KR}$, et al. Acetaminophen-NAPQI hepatotoxicity: a cell line model system genome-wide association study. Toxicol Sci 2011; 120: 33-41.

25 Ohnuma T, Anan E, Hoashi R, Takeda Y, Nishiyama T, Ogura K, et al. Dietary diacetylene falcarindiol induces phase 2 drug-metabolizing enzymes and blocks carbon tetrachloride-induced hepatotoxicity in mice through suppression of lipid peroxidation. Biol Pharm Bull 2011; 34: 371-8.

26 Moffit JS, Aleksunes LM, Kardas MJ, Slitt AL, Klaassen CD, Manautou JE. Role of $\mathrm{NAD}(\mathrm{P}) \mathrm{H}$ : quinone oxidoreductase 1 in clofibrate-mediated hepatoprotection from acetaminophen. Toxicology 2007; 230: 197206.

27 Maher JM, Dieter MZ, Aleksunes LM, Slitt AL, Guo G, Tanaka Y, et al. Oxidative and electrophilic stress induces multidrug resistanceassociated protein transporters via the nuclear factor-E2-related factor-2 transcriptional pathway. Hepatology 2007; 46: 1597-610. 